PROCEEDINGS OF THE

AMERICAN MATHEMATICAL SOCIETY

Volume 130, Number 12, Pages 3537-3544

S 0002-9939(02)06500-0

Article electronically published on May 14, 2002

\title{
COHOMOLOGICAL DIMENSION OF CERTAIN ALGEBRAIC VARIETIES
}

\author{
K. DIVAANI-AAZAR, R. NAGHIPOUR, AND M. TOUSI \\ (Communicated by Wolmer V. Vasconcelos) \\ Dedicated to Professor Hossein Zakeri
}

\begin{abstract}
Let $\mathfrak{a}$ be an ideal of a commutative Noetherian ring $R$. For finitely generated $R$-modules $M$ and $N$ with $\operatorname{Supp} N \subseteq \operatorname{Supp} M$, it is shown that $\operatorname{cd}(\mathfrak{a}, N) \leq \operatorname{cd}(\mathfrak{a}, M)$. Let $N$ be a finitely generated module over a local ring $(R, \mathfrak{m})$ such that $\operatorname{Min}_{\hat{R}} \hat{N}=\operatorname{Assh}_{\hat{R}} \hat{N}$. Using the above result and the notion of connectedness dimension, it is proved that $\operatorname{cd}(\mathfrak{a}, N) \geq \operatorname{dim} N-c(N / \mathfrak{a} N)-$ 1. Here $c(N)$ denotes the connectedness dimension of the topological space Supp $N$. Finally, as a consequence of this inequality, two previously known generalizations of Faltings' connectedness theorem are improved.
\end{abstract}

\section{INTRODUCTION}

Throughout, let $R$ denote a commutative Noetherian ring (with identity) and $\mathfrak{a}$ an ideal of $R$. The study of the cohomological dimension and connectedness of algebraic varieties has produced some interesting results and problems in local algebra. For an $R$-module $M$, the cohomological dimension of $M$ with respect to $\mathfrak{a}$ is defined as

$$
\operatorname{cd}(\mathfrak{a}, M):=\max \left\{i \in \mathbb{Z}: H_{\mathfrak{a}}^{i}(M) \neq 0\right\} .
$$

The cohomological dimension has been studied by several authors; see, for example, Faltings [7, Hartshorne [9] and Huneke-Lyubeznik [1]. In particular in [7] and [1], several upper bounds for cohomological dimension were obtained. The main aim of this article is to establish lower bounds for cohomological dimension of finitely generated modules over a local ring. This is done by using the notion of connectedness dimension. For a Noetherian topological space $X$, the subdimension and connectedness dimension of $X$ are defined respectively as

$$
\begin{aligned}
\operatorname{s} \operatorname{dim} X: & =\min \{\operatorname{dim} Z: Z \text { is an irreducible component of } X\}, \text { and } \\
c(X): & =\min \{\operatorname{dim} Z: Z \subseteq X, Z \text { is closed and } X \backslash Z \text { is disconnected }\} .
\end{aligned}
$$

For more details about these notions, we refer the reader to [3, Ch. 19]. In particular, if $M$ is an $R$-module and $\operatorname{Supp} M$ is considered as a subspace of $\operatorname{Spec} R$ equipped with Zariski topology, we denote $c(\operatorname{Supp} M)$ and $\operatorname{sim}(\operatorname{Supp} M)$ by $c(M)$

Received by the editors October 17, 2000 and, in revised form, August 3, 2001.

2000 Mathematics Subject Classification. Primary 13D45, 14B15.

Key words and phrases. Cohomological dimension, connectedness dimension, subdimension, canonical module.

This research was supported in part by a grant from IPM. 
and $\mathrm{s} \operatorname{dim} M$ respectively. It is clear from the definition that a Noetherian topological space $X$ is connected if and only if $c(X) \geq 0$. Recall that the dimension of the empty space is defined to be -1 .

We shall prove:

Theorem 1.1. Let $(R, \mathfrak{m})$ be a local ring and $N$ a finitely generated $R$-module.

(i) If $R$ is complete, then $\operatorname{cd}(\mathfrak{a}, N) \geq \min \{c(N), \operatorname{sim} N-1\}-c(N / \mathfrak{a} N)$.

(ii) If $\operatorname{Min}_{\hat{R}} \hat{N}=\operatorname{Assh}_{\hat{R}} \hat{N}$, then $\operatorname{cd}(\mathfrak{a}, N) \geq \operatorname{dim} N-c(N / \mathfrak{a} N)-1$.

One of our tools for proving Theorem 1.1 is the following, which plays a key rôle in this paper.

Theorem 1.2. Let $M$ and $N$ be finitely generated $R$-modules with $\operatorname{Supp} N \subseteq$ $\operatorname{Supp} M$. Then $\operatorname{cd}(\mathfrak{a}, N) \leq \operatorname{cd}(\mathfrak{a}, M)$. In particular, $\operatorname{cd}(\mathfrak{a}, N)=\operatorname{cd}(\mathfrak{a}, M)$ whenever $\operatorname{Supp} N=\operatorname{Supp} M$.

In [10], M. Hochster and C. Huneke generalized Faltings' connectedness theorem [6]. Also in [5, P. Schenzel and the first author have proved two generalizations of Faltings' connectedness theorem. As a consequence of Theorem 1.1(ii), we remove the indecomposability condition in [10, Theorem 3.3] and [5, Theorem 4.3].

Our terminology follows that of [5]. Moreover for an $R$-module $M$, the set of minimal elements of $\operatorname{Ass}_{R} M$ is denoted by $\operatorname{Min}_{R} M$ and $\{\mathfrak{p} \in \operatorname{Ass} M: \operatorname{dim} R / \mathfrak{p}=$ $\operatorname{dim} M\}$ by $\operatorname{Assh}_{R} M$.

\section{Cohomological dimension}

First of all, we collect the well known properties of the notion of cohomological dimension in a lemma. Before stating the lemma, recall that the height of an ideal $\mathfrak{a}$ with respect to an $R$-module $M$ is defined as $\operatorname{ht}_{M} \mathfrak{a}=\min \left\{\operatorname{dim} M_{\mathfrak{p}}: \mathfrak{p} \supseteq \mathfrak{a}\right\}$.

Lemma 2.1. Let $\mathfrak{a}$ denote an ideal of $R$. Then:

(i) for an $R$-module $M, \mathrm{ht}_{M} \mathfrak{a} \leq \operatorname{cd}(\mathfrak{a}, M) \leq \operatorname{dim} M$,

(ii) $\operatorname{cd}(\mathfrak{a}, R)=\max \{\operatorname{cd}(\mathfrak{a}, N): N$ is an $R$-module $\}$

$=\max \left\{i \in \mathbb{Z}: H_{\mathfrak{a}}^{i}(N) \neq 0\right.$ for some $R$-module $\left.N\right\}$,

(iii) $\operatorname{cd}(\mathfrak{a}, R) \leq \operatorname{ara}(\mathfrak{a})$, where ara $(\mathfrak{a})$ denotes the arithmetic rank of $\mathfrak{a}$, and

(iv) if $f: R \longrightarrow R^{\prime}$ is a homomorphism of commutative Noetherian rings, then $\operatorname{cd}\left(\mathfrak{a} R^{\prime}, R^{\prime}\right) \leq \operatorname{cd}(\mathfrak{a}, R)$ and, also for any $R^{\prime}$-module $M, \operatorname{cd}(\mathfrak{a}, M)=\operatorname{cd}\left(\mathfrak{a} R^{\prime}, M\right)$. Furthermore if $f$ is faithfully flat, then $\operatorname{cd}\left(\mathfrak{a} R^{\prime}, R^{\prime}\right)=\operatorname{cd}(\mathfrak{a}, R)$.

The following is one of the main results of this paper.

Theorem 2.2. Let $\mathfrak{a}$ denote a proper ideal of $R$ and $M, N$ two finitely generated $R$-modules such that $\operatorname{Supp} N \subseteq \operatorname{Supp} M$. Then $\operatorname{cd}(\mathfrak{a}, N) \leq \operatorname{cd}(\mathfrak{a}, M)$.

Proof. It is enough to show that $H_{\mathfrak{a}}^{i}(N)=0$ for all $i$ with $\operatorname{cd}(\mathfrak{a}, M)<i \leq \operatorname{dim} M+$ 1 , and all finitely generated $R$-module $N$ with $\operatorname{Supp} N \subseteq \operatorname{Supp} M$. We argue this by descending induction on $i$. The assertion is clear for $i=\operatorname{dim} M+1$ by Grothendieck vanishing theorem. Now, suppose $i \leq \operatorname{dim} M$. Since $\operatorname{Supp} N \subseteq$ Supp $M$, by Gruson's theorem [12, Theorem 4.1], there is a chain

$$
0=N_{0} \subset N_{1} \subset N_{2} \subset \cdots \subset N_{k}=N,
$$

such that the factors $N_{j} / N_{j-1}$ are homomorphic images of a direct sum of finitely many copies of $M$. By using short exact sequences, we may reduce the situation to 
the case $k=1$. Then there is an exact sequence

$$
0 \longrightarrow L \longrightarrow M^{n} \longrightarrow N \longrightarrow 0
$$

for some $n \in \mathbb{N}$ and some finitely generated $R$-module $L$. This induces a long exact sequence of local cohomology modules

$$
\ldots \longrightarrow H_{\mathfrak{a}}^{i}(L) \longrightarrow H_{\mathfrak{a}}^{i}\left(M^{n}\right) \longrightarrow H_{\mathfrak{a}}^{i}(N) \longrightarrow H_{\mathfrak{a}}^{i+1}(L) \longrightarrow \ldots,
$$

so that, by the inductive hypothesis, $H_{\mathfrak{a}}^{i+1}(L)=0$. Hence $H_{\mathfrak{a}}^{i}(N)=0$. Thus the argument is complete by induction.

Corollary 2.3. (i) Let $0 \longrightarrow L \longrightarrow M \longrightarrow N \longrightarrow 0$ be an exact sequence of finitely generated $R$-modules. Then $\operatorname{cd}(\mathfrak{a}, M)=\max \{\operatorname{cd}(\mathfrak{a}, L), \operatorname{cd}(\mathfrak{a}, N)\}$.

(ii) Let $f: R \longrightarrow S$ be a monomorphism of commutative Noetherian rings such that $S$ is finitely generated as an $R$-module. Then for any proper ideal $\mathfrak{a}$ of $R$, $\operatorname{cd}(\mathfrak{a}, R)=\operatorname{cd}(\mathfrak{a} S, S)$.

(iii) If $M$ is a finitely generated faithful $R$-module, then $\operatorname{cd}(\mathfrak{a}, M)=\operatorname{cd}(\mathfrak{a}, R)$.

Proof. (i) From the long exact sequence

$$
\ldots \longrightarrow H_{\mathfrak{a}}^{i}(L) \longrightarrow H_{\mathfrak{a}}^{i}(M) \longrightarrow H_{\mathfrak{a}}^{i}(N) \longrightarrow H_{\mathfrak{a}}^{i+1}(L) \longrightarrow \ldots,
$$

we deduce $\operatorname{cd}(\mathfrak{a}, M) \leq \max \{\operatorname{cd}(\mathfrak{a}, L), \operatorname{cd}(\mathfrak{a}, N)\}$, while Theorem 2.2 implies $\max \{\operatorname{cd}(\mathfrak{a}, L), \operatorname{cd}(\mathfrak{a}, N)\} \leq \operatorname{cd}(\mathfrak{a}, M)$. Therefore (i) holds.

(ii) follows by Lemma 2.1(iv) and Theorem 2.2.

(iii) Clearly $\operatorname{Supp} M=\operatorname{Spec} R$, and so the result follows by Theorem 2.2.

Remark 2.4. (i) One can deduce Lemma 2.1(ii) from Theorem 2.2 easily, because $H_{\mathfrak{a}}^{i}(\cdot)$ commutes with direct limits.

(ii) Part (ii) of Corollary 2.3 is proved in [9, Proposition 2.1] by using methods of algebraic geometry.

(iii) Let $M$ and $N$ be two finitely generated $R$-modules such that $M \neq \mathfrak{a} M$ and that $\operatorname{Supp}\left(N / \Gamma_{\mathfrak{a}}(N)\right) \subseteq \operatorname{Supp}\left(M / \Gamma_{\mathfrak{a}}(M)\right)$. Then $\operatorname{cd}(\mathfrak{a}, N) \leq \operatorname{cd}(\mathfrak{a}, M)$.

(iv) Let $M$ and $N$ be two finitely generated $R$-modules. For each $i \in \mathbb{N}_{0}$,

$$
\max \left\{\operatorname{cd}\left(\mathfrak{a}, \operatorname{Ext}_{R}^{i}(M, N)\right), \operatorname{cd}\left(\mathfrak{a}, \operatorname{Tor}_{i}^{R}(M, N)\right)\right\} \leq \min \{\operatorname{cd}(\mathfrak{a}, M), \operatorname{cd}(\mathfrak{a}, N)\} .
$$

(v) In view of Corollary 2.3(iii) results concerning cohomological dimension of $R$ with respect to an ideal $\mathfrak{a}$ can be extended to $\operatorname{cd}(\mathfrak{a}, M)$ for any finitely generated faithful $R$-module $M$. See for example [4, Theorem 2 and Remark].

We shall use the following result in the proof of Theorem 2.7.

Lemma 2.5. Let the situation be as in Lemma 2.1, and let $x \in R$. Then for an R-module $M$,

$$
\operatorname{cd}(\mathfrak{a}+R x, M) \leq \operatorname{cd}(\mathfrak{a}, M)+1 .
$$

Proof. Let $\mathfrak{b}=\mathfrak{a}+R x$ and $\operatorname{cd}(\mathfrak{a}, M)=r$. By [3, Proposition 8.1.2], there is a long exact sequence

$$
\ldots \longrightarrow H_{\mathfrak{b}}^{i}(M) \longrightarrow H_{\mathfrak{a}}^{i}(M) \longrightarrow H_{\mathfrak{a}}^{i}\left(M_{x}\right) \longrightarrow H_{\mathfrak{b}}^{i+1}(M) \longrightarrow H_{\mathfrak{a}}^{i+1}(M) \longrightarrow \ldots
$$

where $M_{x}$ is the localization of $M$ with respect to the multiplicatively closed subset $\left\{x^{i}: i \in \mathbb{N}_{0}\right\}$ of $R$. Since $H_{\mathfrak{a}}^{i}(M)=0$ for all $i>r$, it turns out that $H_{\mathfrak{a}}^{i}\left(M_{x}\right) \cong$ $H_{\mathfrak{b}}^{i+1}(M)$ for all $i>r$. Thus each element of $H_{\mathfrak{a}}^{i}\left(M_{x}\right)$ is annihilated by some power of $\mathfrak{b}$. By applying the functor $H_{\mathfrak{a}}^{i}(\cdot)$ on the isomorphism $M_{x} \stackrel{x^{n}}{\longrightarrow} M_{x}, n \in \mathbb{N}$, we 
deduce that $H_{\mathfrak{a}}^{i}\left(M_{x}\right) \stackrel{x^{n}}{\longrightarrow} H_{\mathfrak{a}}^{i}\left(M_{x}\right)$ is an isomorphism. But each element of $H_{\mathfrak{a}}^{i}\left(M_{x}\right)$ is annihilated by $x^{n}$ for some $n \in \mathbb{N}$. This yields that $H_{\mathfrak{a}}^{i}\left(M_{x}\right)=0$ for all $i>r$. Therefore $H_{\mathfrak{b}}^{i}(M)=0$ for all $i>r+1$, as required.

We recall some properties of the notions $c(N)$ and $\mathrm{s} \operatorname{dim} N$ in the following lemma (see [3, Ch. 19]).

Lemma 2.6. Let $N$ be a finitely generated $R$-module. Then the following hold:

(i) $\operatorname{s} \operatorname{dim} N=\min \left\{\operatorname{dim} R / \mathfrak{p}: \mathfrak{p} \in \operatorname{Min}_{R} N\right\}$,

(ii) $c(N)=\min \left\{\operatorname{dim}\left(R /\left(\bigcap_{\mathfrak{p} \in A} \mathfrak{p}+\bigcap_{\mathfrak{p} \in B} \mathfrak{p}\right)\right): A\right.$ and $B$ are non-empty subsets of $\operatorname{Min}_{R} N$ such that $\left.A \cup B=\operatorname{Min}_{R} N\right\}$,

(iii) $c(N) \leq \mathrm{s} \operatorname{dim} N$, and

(iv) if $(R, \mathfrak{m})$ is local, then $c(\operatorname{Supp} N \backslash\{\mathfrak{m}\})=c(N)-1$.

Theorem 2.7. Let $\mathfrak{a}, \mathfrak{b}$ be two ideals of a local ring $(R, \mathfrak{m})$ and $N$ a finitely generated $R$-module such that $\min \{\operatorname{dim} N / \mathfrak{a} N, \operatorname{dim} N / \mathfrak{b} N\}>\operatorname{dim} N /(\mathfrak{a}+\mathfrak{b}) N$.

(i) If $\operatorname{Min}_{\hat{R}} \hat{N}$ consists of a single prime $\mathfrak{p}$, then

$$
\operatorname{cd}(\mathfrak{a} \cap \mathfrak{b}, N) \geq \operatorname{dim} N-\operatorname{dim} N /(\mathfrak{a}+\mathfrak{b}) N-1 .
$$

(ii) If $R$ is complete, then

$$
\operatorname{cd}(\mathfrak{a} \cap \mathfrak{b}, N) \geq \min \{c(N), \operatorname{sim} N-1\}-\operatorname{dim} N /(\mathfrak{a}+\mathfrak{b}) N .
$$

Proof. Let $R_{1}=R / \operatorname{Ann}_{R} N$. Then $\operatorname{cd}(\mathfrak{a} \cap \mathfrak{b}, N)=\operatorname{cd}\left((\mathfrak{a} \cap \mathfrak{b}) R_{1}, R_{1}\right)$, by Lemma 2.1(iv) and Theorem 2.2. On the other hand one can easily check that $\mathrm{s} \operatorname{dim} N=$ $\mathrm{s} \operatorname{dim} R_{1}$ and that $c(N)=c\left(R_{1}\right)$. Therefore we may and do assume that $N=$ $R$. Now, by replacing $\operatorname{ara}(\mathfrak{a} \cap \mathfrak{b})$ by $\operatorname{cd}(\mathfrak{a} \cap \mathfrak{b}, R)$ and using Lemma 2.5 , we can process similar to the proof of [3, Proposition 19.2.7] to deduce (i). Also, in view of Lemma 2.1(i) and 2.1(iv), one can deduce (ii) by similar argument as in $\underline{3}$, Lemma 19.2.8].

Now, we are ready to state the next main theorem of this section, namely the connectedness bound for a finitely generated module over a complete local ring which is a generalization and refinement of Grothendieck's connectedness theorem (see [8, Expose XIII, Théorém 2.1]).

Theorem 2.8. Let $\mathfrak{a}$ be a proper ideal of a complete local ring $(R, \mathfrak{m})$, and let $N$ be a finitely generated $R$-module. Then

$$
\operatorname{cd}(\mathfrak{a}, N) \geq \min \{c(N), \operatorname{sim} N-1\}-c(N / \mathfrak{a} N) .
$$

Proof. Let $\operatorname{Min}_{R}(N / \mathfrak{a} N)=\left\{\mathfrak{p}_{1}, \ldots, \mathfrak{p}_{n}\right\}$ and $c:=c(N / \mathfrak{a} N)$. If $n=1$, we have $c=\operatorname{dim} R / \mathfrak{p}_{1}$ (see Lemma 2.6(ii)). Let $\mathfrak{p} \in \operatorname{Min}_{R} N$ be such that $\mathfrak{p} \subseteq \mathfrak{p}_{1}$. Then as Supp $R / \mathfrak{p} \subseteq \operatorname{Supp} N$, by virtue of Lemmas 2.1(i), 2.1(iv) and Theorem 2.2,

$$
\text { ht } \mathfrak{p}_{1} / \mathfrak{p} \leq \operatorname{cd}\left(\mathfrak{p}_{1} / \mathfrak{p}, R / \mathfrak{p}\right)=\operatorname{cd}\left(\mathfrak{p}_{1}, R / \mathfrak{p}\right) \leq \operatorname{cd}\left(\mathfrak{p}_{1}, N\right) .
$$

Because $\operatorname{Rad}\left(\mathfrak{a}+\operatorname{Ann}_{R} N\right)=\mathfrak{p}_{1}$, it turns out that $\operatorname{cd}\left(\mathfrak{p}_{1}, N\right)=\operatorname{cd}(\mathfrak{a}, N)$.

Next, since $R / \mathfrak{p}$ is catenary, we deduce that $c=\operatorname{dim} R / \mathfrak{p}-\operatorname{ht} \mathfrak{p}_{1} / \mathfrak{p} \geq \operatorname{sim} N-$ $\operatorname{cd}(\mathfrak{a}, N)$, as desired. Accordingly, we may assume that $n>1$. Then there exist two non-empty subsets $A, B$ of $\operatorname{Min}_{R} N / \mathfrak{a} N$ for which $A \cup B=\operatorname{Min}_{R} N / \mathfrak{a} N$, and

$$
c=\operatorname{dim}\left(R /\left(\bigcap_{\mathfrak{p} \in A} \mathfrak{p}\right)+\left(\bigcap_{\mathfrak{p} \in B} \mathfrak{p}\right)\right)
$$


Moreover, we may assume that $A \cap B=\emptyset$. Put $\mathfrak{b}:=\bigcap_{\mathfrak{p} \in A} \mathfrak{p}$ and $\mathfrak{c}:=\bigcap_{\mathfrak{p} \in B} \mathfrak{p}$. Then $\operatorname{dim} N / \mathfrak{b} N>c, \operatorname{dim} N / \mathfrak{c} N>c$ and $\mathfrak{b} \cap \mathfrak{c}=\operatorname{Rad}\left(\mathfrak{a}+\operatorname{Ann}_{R} N\right)$. Therefore the proof finishes by Theorem 2.7(ii).

Corollary 2.9. Let the situation be as in Theorem 2.8. Then $\operatorname{cd}(\mathfrak{a}, N) \geq c(N)-$ $c(N / \mathfrak{a} N)-1$. Moreover if $\left|\operatorname{Min}_{R} N\right|>1$, then the inequality is strict.

Proof. The assertion is clear by Theorem 2.8, because, by Lemma 2.6(iii), $c(N) \leq$ $\mathrm{s} \operatorname{dim} N$, with strict inequality if $\left|\operatorname{Min}_{R} N\right|>1$.

\section{Connectedness theorem}

In [10, M. Hochster and C. Huneke have extended Faltings' original connectedness theorem [6] as follows. Let $(R, \mathfrak{m})$ be an equidimensional complete local ring of dimension $d$, and $\mathfrak{a}$ a proper ideal of $R$. If $H_{\mathfrak{m}}^{d}(R)$ is indecomposable, then the punctured spectrum of $R / \mathfrak{a}$ is connected provided $\operatorname{cd}(\mathfrak{a}, R) \leq d-2$. Next this result is generalized to finitely generated modules in [5]. In this section, our objective is to remove the indecomposability assumption. To this end, we give a refinement of Theorem 2.8 in Theorem 3.4. Before we do this, we bring some definitions and lemmas.

Definition. Let $(R, \mathfrak{m})$ be a $d$-dimensional local ring. $A$ finitely generated $R$ module $K$ is called the canonical module of $R$, if $K \otimes_{R} \hat{R} \cong \operatorname{Hom}_{R}\left(H_{\mathfrak{m}}^{d}(R), E(R / \mathfrak{m})\right)$.

Proposition 3.1. Let $\mathfrak{p}_{1}, \ldots, \mathfrak{p}_{n}$ be prime ideals of a finite dimensional Noetherian ring $R$ such that $\mathfrak{p}_{i} \nsubseteq \mathfrak{p}_{j}$ for all $1 \leq i \neq j \leq n$. Suppose that $R$ is $\left(S_{2}\right)$ and that $R_{\mathfrak{p}}$ possesses a canonical module for all $\mathfrak{p} \in \operatorname{Spec} R$. Also, assume that for each prime ideal $\mathfrak{p}$ of $R, \operatorname{dim} R=\operatorname{dim} R / \mathfrak{p}+$ htp. Set $\mathfrak{a}:=\bigcap_{i=1}^{m} \mathfrak{p}_{i}$ and $\mathfrak{b}=\bigcap_{i=m+1}^{n} \mathfrak{p}_{i}$ for some $1 \leq m<n$. Then

$$
\operatorname{cd}(\mathfrak{a} \cap \mathfrak{b}, R) \geq \operatorname{dim} R-\operatorname{dim} R /(\mathfrak{a}+\mathfrak{b})-1 .
$$

Proof. Let $\mathfrak{q}$ be a prime ideal of $R$ containing $\mathfrak{a}+\mathfrak{b}$ such that $\operatorname{dim} R /(\mathfrak{a}+\mathfrak{b})=$ $\operatorname{dim} R / \mathfrak{q}$. Our assumption on $\mathfrak{p}_{i}$ 's implies that the ideals $\mathfrak{a} R_{\mathfrak{q}}$ and $\mathfrak{b} R_{\mathfrak{q}}$ are not $\mathfrak{q} R_{\mathfrak{q}}$-primary. Now the claim follows immediately from Lemma 2.1(iv) and the following lemma.

Lemma 3.2. Let $(R, \mathfrak{m})$ be a $\left(S_{2}\right)$ local ring which possesses a canonical module. Let $\mathfrak{a}$ and $\mathfrak{b}$ be two non-m-primary ideals of $R$ such that $\mathfrak{a}+\mathfrak{b}$ is $\mathfrak{m}$-primary. Then

$$
\operatorname{cd}(\mathfrak{a} \cap \mathfrak{b}, R) \geq \operatorname{dim} R-1 .
$$

Proof. Assume that the contrary is true. Then the Mayer-Vietoris sequence (see e.g. [3, 3.2.3]) yields the isomorphism

$$
H_{\mathfrak{m}}^{d}(R)=H_{\mathfrak{a}+\mathfrak{b}}^{d}(R) \cong H_{\mathfrak{a}}^{d}(R) \oplus H_{\mathfrak{b}}^{d}(R)
$$

The module $H_{\mathfrak{m}}^{d}(R)$ is indecomposable by [2, Remark 1.4] and so either $H_{\mathfrak{a}}^{d}(R)=0$ or $H_{\mathfrak{b}}^{d}(R)=0$. Suppose $H_{\mathfrak{b}}^{d}(R)=0$; then $H_{\mathfrak{m}}^{d}(R) \cong H_{\mathfrak{a}}^{d}(R)$. It follows from [2, Proposition 1.2 and Lemma 1.1] that Assh $\hat{R}=$ Ass $\hat{R}$. By virtue of [3, Ex. 8.2.6], once applied to $\mathfrak{m}$ and a second time applied to $\mathfrak{a}$, it follows that $\operatorname{dim} \hat{R} / \mathfrak{a} \hat{R}+\mathfrak{p}=$ 0 for all $\mathfrak{p} \in \operatorname{Ass} \hat{R}$. This leads that $\mathfrak{a}$ is $\mathfrak{m}$-primary, which is a contradiction. 
Lemma 3.3. Let $R$ be a Noetherian ring such that $R$ is $\left(S_{2}\right)$ and that $R_{\mathfrak{p}}$ has a canonical module for all $\mathfrak{p} \in \operatorname{Spec} R$. Assume that $\operatorname{dim} R$ is finite and that for each $\mathfrak{p} \in \operatorname{Spec} R, \operatorname{dim} R=\operatorname{dim} R / \mathfrak{p}+$ ht $\mathfrak{p}$. Then for each proper ideal $\mathfrak{a}$ of $R$,

$$
\operatorname{cd}(\mathfrak{a}, R) \geq \operatorname{dim} R-c(R / \mathfrak{a})-1 .
$$

Proof. Without loss of generality we can and do assume that $\mathfrak{a}=\operatorname{Rad}(\mathfrak{a})$. Let $\mathfrak{p}_{1}, \ldots, \mathfrak{p}_{n}$ be the distinct minimal primes of $\mathfrak{a}$, and let $c:=c(R / \mathfrak{a})$. If $n=1$, we have $\mathfrak{a}=\mathfrak{p}_{1}$ and $c=\operatorname{dim} R / \mathfrak{p}_{1}$. Hence

$$
c=\operatorname{dim} R-\operatorname{ht} \mathfrak{p}_{1} \geq \operatorname{dim} R-\operatorname{cd}\left(\mathfrak{p}_{1}, R\right) .
$$

Consider now the case where $n>1$. By Lemma 2.6(ii), there exist two disjoint non-empty subsets $A, B$ of $\{1, \ldots, n\}$ for which $A \cup B=\{1, \ldots, n\}$ and $c=$ $\operatorname{dim}\left(R /\left(\bigcap_{i \in A} \mathfrak{p}_{i}\right)+\left(\bigcap_{j \in B} \mathfrak{p}_{j}\right)\right)$. Set $\mathfrak{b}=\bigcap_{i \in A} \mathfrak{p}_{i}$ and $\mathfrak{c}=\bigcap_{j \in B} \mathfrak{p}_{j}$. Then $\mathfrak{p}_{i} \not \subset \mathfrak{p}_{j}$ for all $1 \leq i, j \leq n$, and $\mathfrak{b} \cap \mathfrak{c}=\mathfrak{a}$. We can now use Proposition 3.1 to complete the proof.

Theorem 3.4. Let $\mathfrak{a}$ be a proper ideal of a local ring $(R, \mathfrak{m})$ and let $N$ be a finitely generated $R$-module such that $\operatorname{Min}_{\hat{R}} \hat{N}=\operatorname{Assh}_{\hat{R}} \hat{N}$. Then

$$
\operatorname{cd}(\mathfrak{a}, N) \geq \operatorname{dim} N-c(N / \mathfrak{a} N)-1 .
$$

Proof. Let $R_{1}=R / \operatorname{Ann}_{R} N$. Then $c(N / \mathfrak{a} N)=c\left(R_{1} / \mathfrak{a} R_{1}\right)$ and $\operatorname{cd}(\mathfrak{a}, N)=$ $\operatorname{cd}\left(\mathfrak{a} R_{1}, R_{1}\right)$ by Lemma 2.1(iv) and Theorem 2.2. On the other hand $\operatorname{Min} \hat{R}_{1}=$ Assh $\hat{R}_{1}$. Thus it is sufficient to prove the claim for the ring $R$ itself. Since $c(R / \mathfrak{a}) \geq c(\hat{R} / \mathfrak{a} \hat{R})$ by [3 Lemma 19.3.1], we can assume that $R$ is complete. Since $\mathrm{s} \operatorname{dim} R=\operatorname{dim} R$, in view of Theorem 2.8 it is enough to show that $c(R) \geq \operatorname{dim} R-1$. Let $J=\bigcap \mathfrak{q}$, where $\mathfrak{q}$ runs through all the primary components of the zero ideal of $R$ such that $\operatorname{dim} R / \mathfrak{q}=\operatorname{dim} R$. It is clear that $\operatorname{dim} R / J=\operatorname{dim} R$. Also, since Min $R=\operatorname{Assh} R$, it follows from Lemma 2.6(ii) that $c(R / J)=c(R)$. Thus by replacing $R$ with $R / J$, we may assume that Assh $R=$ Ass $R$. By [1 1.11 and Theorem 3.2], there exists a commutative Noetherian semi-local $\operatorname{ring} S$ and a monomorphism $\varphi: R \longrightarrow S$ such that:

(i) $S$ is finitely generated as an $R$-module,

(ii) $S$ is $\left(S_{2}\right)$,

(iii) $S_{\mathfrak{p}}$ has a canonical module for all $\mathfrak{p} \in \operatorname{Spec} S$, and

(iv) every maximal chain of prime ideals in $S$ is of length $\operatorname{dim} S$.

Let $\mathfrak{p}_{1}, \ldots, \mathfrak{p}_{n}$ be the distinct minimal prime ideals of $R$. Then there exist two non-empty subsets $A, B$ of $\{1, \ldots, n\}$ for which $A \cup B=\{1, \ldots, n\}$ and

$$
c(R)=\operatorname{dim}\left(R /\left(\bigcap_{i \in A} \mathfrak{p}_{i}\right)+\left(\bigcap_{j \in B} \mathfrak{p}_{j}\right)\right) .
$$

Since by condition (i), $S$ is integral over $R$, it follows that $\operatorname{dim} R=\operatorname{dim} S$ and that for each $1 \leq i \leq n$ there exists $\mathfrak{q}_{i} \in \operatorname{Spec} S$ such that $\varphi^{-1}\left(\mathfrak{q}_{i}\right)=\mathfrak{p}_{i}$. For a given prime ideal $\mathfrak{q}$ of $S$, we show that $\mathfrak{q} \in \operatorname{Min} S$ if and only if $\mathfrak{p}=\varphi^{-1}(\mathfrak{q}) \in \operatorname{Min} R$. To this end, first note that the ring $S / \mathfrak{q}$ is integral over the $\operatorname{ring} R / \mathfrak{p}$, and so $\operatorname{dim} S / \mathfrak{q}=\operatorname{dim} R / \mathfrak{p}$. Since Ass $R=\operatorname{Assh} R$, it turns out that $\mathfrak{p} \in \operatorname{Min} R$ if and only if $\operatorname{dim} R / \mathfrak{p}=\operatorname{dim} R$. On the other hand (iv) implies that $\mathfrak{q} \in \operatorname{Min} S$ if and only if 
$\operatorname{dim} S / \mathfrak{q}=\operatorname{dim} S$. Therefore the claim is immediate. Put

$$
A^{\prime}=\left\{\mathfrak{q} \in \operatorname{Min} S: \varphi^{-1}(\mathfrak{q})=\mathfrak{p}_{i} \text { for some } i \in A\right\}
$$

and $B^{\prime}=\left\{\mathfrak{q} \in \operatorname{Min} S: \varphi^{-1}(\mathfrak{q})=\mathfrak{p}_{j}\right.$ for some $\left.j \in B\right\}$. So, we have

$$
\begin{aligned}
c(R) & \geq \operatorname{dim} R / \varphi^{-1}\left(\left(\bigcap_{\mathfrak{q} \in A^{\prime}} \mathfrak{q}\right)+\left(\bigcap_{\mathfrak{q} \in B^{\prime}} \mathfrak{q}\right)\right) \\
& =\operatorname{dim} S /\left(\bigcap_{\mathfrak{q} \in A^{\prime}} \mathfrak{q}+\bigcap_{\mathfrak{q} \in B^{\prime}} \mathfrak{q}\right) \geq c(S) .
\end{aligned}
$$

Therefore the result follows by Lemma 3.3. Note that by (iv), for each prime ideal $\mathfrak{p}$ of $S, \operatorname{dim} S=\operatorname{dim} S / \mathfrak{p}+$ ht $\mathfrak{p}$.

Now we are prepared to present the main result of this section which is a generalization of [10, Theorem 3.3] and of [5, Corollary 4.2 and Theorem 4.3].

Corollary 3.5. Let $\mathfrak{a}$ be a proper ideal of a local ring $(R, \mathfrak{m})$. Let $N$ be a $d$ dimensional finitely generated R-module such that $\operatorname{Assh}_{\hat{R}} \hat{N}=\operatorname{Min}_{\hat{R}} \hat{N}$. Then $\operatorname{Supp} N / \mathfrak{a} N \backslash\{\mathfrak{m}\}$ is connected provided $\operatorname{cd}(\mathfrak{a}, N) \leq d-2$.

Proof. By Lemma 2.6(iv), $c(\operatorname{Supp}(N / \mathfrak{a} N) \backslash\{\mathfrak{m}\})=c(N / \mathfrak{a} N)-1$. Hence by Theorem $3.4, c(\operatorname{Supp}(N / \mathfrak{a} N) \backslash\{\mathfrak{m}\}) \geq \operatorname{dim} N-\operatorname{cd}(\mathfrak{a}, N)-2$. Thus

$$
c(\operatorname{Supp}(N / \mathfrak{a} N) \backslash\{\mathfrak{m}\}) \geq 0,
$$

and so $\operatorname{Supp}(N / \mathfrak{a} N) \backslash\{\mathfrak{m}\}$ is connected, as desired.

\section{REFERENCES}

[1] Y. Aoyama, Some basic results on canonical modules, J. Math. Kyoto Univ., 23-1 (1983), 85-94. MR 84i:13015

[2] Y. Aoyama, S.Goto, On the endomorphism ring of the canonical module, J. Math. Kyoto Univ., 25-1 (1985), 21-30. MR 86e:13021

[3] M. Brodmann, R. Y. Sharp: 'Local cohomology-An algebraic introduction with geometric applications', Cambr. Univ. Press, 1998. MR 99h:13020

[4] D. Delfino, A vanishing theorem for local cohomology modules, J. Pure. Appl. Algebra 115 (1997), 107-111. MR 98f:13015

[5] K. Divaani-Aazar, P. Schenzel, Ideal topologies, local cohomology and connectedness, Math. Proc. Camb. Phil. Soc 131 (2001), 211-226. CMP 2002:01

[6] G. Faltings, Some theorems about formal functions, Publ. of R.I.M.S. Kyoto 16 (1980), 721737. MR 82h:14004

[7] G. Faltings, Über lokale Kohomologiegruppen hoher Ordnung, J. Reine Angew. Math. 313 (1980), 43-51. MR 82f:14006

[8] A. Grothendieck, Cohomologie local des faisceaux coherents et theorems de Lefschetz locaux et globaux (SGA2), Seminaire de Geometrie Algebrique du Bois Marie 1962 (North-Holland, Amsterdam 1968). MR 57:16294

[9] R. Hartshorne, Cohomological dimension of algebraic varieties, Annals of Math. 88 (1968), 403-450. MR 38:1103

[10] M. Hochster, C. Huneke, Indecomposable canonical modules and connectedness, Contemporary Mathematics 159 (1994), 197-208. MR 95e:13014 
[11] C. Huneke, G. Lyubeznik, On the vanishing of local cohomology modules, Inv. Math. 102 (1990), 73-93. MR 91i:13020

[12] W. Vasconcelos, Divisor theory in module categories (North-Holland, Amsterdam, 1974). MR 58:16637

Institute for Studies in Theoretical Physics and Mathematics, P.O. Box 19395-5746, Tehran, Iran - and - Department of Mathematics, Az-Zahra University, Tehran, Iran

E-mail address: kdivaani@ipm.ir

Institute for Studies in Theoretical Physics and Mathematics, P.O. Box 19395-5746, Tehran, Iran - and - Department of Mathematics, University of Tabriz, Tabriz, Iran

E-mail address: naghipour@tabrizu.ac.ir

Institute for Studies in Theoretical Physics and Mathematics, P.O. Box 19395-5746, Tehran, Iran - and - Department of Mathematics, Shahid Beheshti University, Tehran, IRAN

E-mail address: mtousi@vax.ipm.ac.ir 